\title{
Personal experience with self-care training in different settings
}

\author{
Linda Lehman ${ }^{\mathrm{a}}$ \\ ${ }^{a}$ Retired American Leprosy Missions, Rio Rancho, New Mexico, USA \\ e-mail:Ifayelehman@gmail.com
}

Submitted and accepted 17 November 2021

\begin{abstract}
Summary This is a personal account of my experience with training, care and self-care practices within Neglected Tropical Diseases and other diseases and health conditions. Forty plus years of experience, led to the development of The Ten Steps: A guide for health promotion and empowerment of people affected by Neglected Tropical Diseases. Successful NTD disease control programs not only control the disease within a population but ensure that a person-centered continuum of care is developed. Prioritizing the teaching of self-care and involving the person, the family and caregiver in care is critical to sustaining care at home. Briefly, some important lessons are shared about training, identifying, and treating problems early and the importance of the participation of the person affected, their family and caregiver in care. The biggest surprises to health workers were how much improvement can be achieved through very simple interventions, and the positive results of involving the person affected.
\end{abstract}

Keywords: Self-care, care, NTDs, health promotion, empowerment, training, participation

\section{Introduction}

The following article relates to my professional work as an Occupational Therapist and Public Health Worker providing and teaching care to health care workers (HCW), persons affected and their families at home, in the community and at all levels of health care services in a global context across many health conditions and diseases. The strong desire to be able to participate in family, school, work, and community life, motivates an individual to learn selfcare. However, the strong desire to be included and to participate can also cause the person to ignore best self-care practices. It is for this reason that the support of family, friends, health workers, work colleagues and community are important to enabling best self-care practices.

The Ten Steps: A guide for health promotion and empowerment of people affected by Neglected Tropical Diseases (NTDs) ${ }^{1}$ evolved from these experiences and reflects more than 40 years of collective wisdom from Linda F. Lehman, Mary Jo Geyer and Laura Bolton. The guide provides knowledge and practical skills used to address common physical problems 
faced in skin NTDs but also in other diseases and health conditions. Early identification and actions to manage the problem frequently had an immediate positive result such as improving wound healing, reducing pain, improving mobility and hand function, and increasing participation. Recommended care was based on evidence-based practices or best practices across many diseases and health conditions. Teaching HCW to facilitate and coach self-care practices contributes to decreasing the person's sense of "helplessness" and improves the person's ownership and participation in health practices. Person/family/community centered problem solving uses locally available resources and learns when to seek additional help. The three-part guide includes a summary card of frequent care issues with basic care actions, an executive summary for program managers and an extensive guide for participatory training with references, activities, handouts, and lists of equipment and materials needed for training.

The biggest difference between care in NTDs and other health conditions and diseases is that most people affected by NTDs live in poor, resource limited environments with difficult access to water, sanitation, transportation, education, work, and health services. Teaching persons affected to recognize and successfully manage common problems is essential to maintaining health and coping with disease and disability with or without a health worker.

\section{Important lessons learned}

This section highlights some of the important issues learned in three areas: training, identifying problems early and taking action, and participation of the person/family/caregiver in care.

\section{TRAINING}

Frequently NTD training focused on how to identify and treat the disease and supervise disease control documentation with little time dedicated to teaching early detection and management of complications. Training was often done using lectures, PowerPoint presentations and discussions. Often training was done top-down by professional staff-from doctor, nurse to community health worker. As a result, health teams had difficulty working together as a team, communicating with each other, and using a common consistent language that was easily understood by the person affected, their families and caregivers. Trainers often had good theoretical knowledge but limited practical knowledge, skills, and experience with best practice or evidence-based care. Training did not address how to identify and address small problems or impairments that often would develop into bigger complications because no action was taken. The most frequent physical problems noted in skin NTDs were wounds, scars, edema, and peripheral neuropathy causing motor and sensory loss. These problems frequently would limit movement and mobility and restrict the person's participation in family, school, work, and community life as well as cause stigmatizing visible reminders of their disease.

The most important lesson learned in training was the importance of including persons affected with or without family or caregivers within the training. Training participants always commented that they learned the most from the practice with patients. Many HCW repeatably stated they were surprised how starting care early could improve conditions so quickly. They further stated by doing and seeing this practice gave them the knowledge, skills, confidence which motivated them to start care interventions earlier and to teach self-care.

The Ten Steps Guide was set up to address the most frequent physical problems and to improve care within NTD training, treatment, and supervision activities. It encouraged participatory methods of training and training by health teams which included the doctor, nurse, community health worker and district disease control officer. Improvements were then 
observed in the team's communication and collaboration, as well as improvements in the support from the disease control officer to the team. District disease control officers stated that participation helped them understand better how to check on care-giving within their own monitoring and evaluation activities, and provide further on-the-job training, as needed.

\section{IDENTIFY PROBLEMS EARLY AND TAKING ACTION}

Visiting homes, communities and all levels of health care facilities revealed that many problems were not recognized early or they were thought to be "small problems" and had no need for daily care. In Buruli ulcer sometimes 25-30\% of movement limitations were not identified. When the HCW and person affected learned to compare the affected side with unaffected side they identified both the movement limitation and edema which led to early actions to address the problem.

Some interventions, like wound management, if not managed well, caused bigger problems. An example was tight bandages restricting movement and causing edema. If wounds were at or near a joint, the person was at great risk of developing contractures. This required that movement be done when bandages were removed as well as using unrestrictive bandaging allowing movement to be done throughout the day. In addition, positioning limbs in opposition to the contracting forces of wound healing had to be done at rest and at night to prevent contractures. Children responded with less fear and more interest when they were allowed to participate in wound care, and exercises were adapted within play and daily activities. To accomplish this, the person affected, family and caregivers needed to learn what to do on a daily basis. The HCW and other people affected were key to teaching them.

Some delays in care were the result of unhelpful attitudes and beliefs. For example, some felt movement could not be done if there was a wound or that movement limitations could only be addressed at a distant reference health service by a specialist in rehabilitation. Delays in action and/or inadequate management resulted in greater impairments and limitations, increasing disability and stigma. This affected mental well-being and ability to participate in family, school, and socioeconomic activities.

The Ten Steps Guide aimed to help the HCW know what simple actions to take to care for eyes, skin and nails, wounds, scars, edema, movement limitations, and how to protect eyes, hands, and feet at risk of injury due to sensory loss. They also learned when care should be referred. Monitoring and evaluation activities are key to assuring adequate care and timely referral is done. Successful NTD programs included both disease control and the continuum of care. ${ }^{2}$

\section{PARTICIPATION OF PERSON AFFECTED/FAMILY/CAREGIVER IN CARE}

$\mathrm{HCW}$, at all levels and surprisingly at referral hospitals, frequently did not include the participation of the person affected, their family and caregiver in care activities. Sometimes HCW felt care was the responsibility of only the HCW, sometimes the HCW complained that there was not enough time to teach the person, their family or caregiver. Hospitals stays and hospital discharges were made with the person never learning how to do their own self-care.

The Ten Steps Guide helped the HCW learned how to do simple interventions at the bedside, during wound care and involve the person, family and caregiver. HCW learned the importance of giving family and caregivers permission and coaching them on how to help the affected person to move, to participate in doing daily activities, to play, and know how to position during rest and at night. Empowerment in self-care practices lessened fears and the sense of "helplessness" during hospital stays and health clinic visits. Learning self-care during hospital 
stays was important for sustaining the continuation of care at home. It enabled individuals of all ages to return to their daily life activities, fulfill meaningful life roles and maximize their wellbeing. Individual self-care practices and developing self-care groups empowered the person to obtain and sustain improved function and social inclusion.

\section{Conclusions}

Successful NTD disease control programs not only control the disease within a population but ensure that a person-centered continuum of care is developed. Prioritizing the teaching of self-care and involving the person, the family and caregiver in care is critical to sustaining care at home. The development of self-care and self-help groups within the community adds additional benefits of problem-solving issues together to address barriers limiting inclusion and providing important psychosocial support. Self-care, access to assistive technology and functioning referral systems are the keys to enabling the person to maximize their function and enabling them to fully participate in family, school, work and community life.

The biggest surprise of health workers who get seriously involved in self-care training is how much improvement, on many levels, can be achieved through very simple interventions. The question for program managers, governmental and non-governmental organizations is why these simple interventions are not done, or were not started earlier, and what can they do to assure self-care practices are included.

There is a need for well-designed training sessions which mix different levels of health workers and include persons affected/caregivers to practice doing the interventions and stimulating self-care practices. This requires that sufficient time and resources are planned to enable the practice of interventions and self-care during training. It may also require further informal "on the job" training during routine monitoring visits.

The HCW learn that they do not need to be "in charge" of all care, but can task share and support persons affected/caregivers in self-care activities in the hospital, at home and in the community. It is essential that the HCW give persons affected and care givers "permission" and enable them to practice daily self-care. Self-care is a key practice for alleviating suffering by managing morbidity and preventing disability in NTDs over the continuum of care. Is selfcare included in NTD elimination certifications? Inclusion in the certification process would strongly communicate that self-care practices are essential for true elimination.

\section{References}

1 Lehman LF, Geyer MJ, Bolton L. Ten steps: A guide for health promotion and empowerment of people affected by neglected tropical diseases. Greenville: American Leprosy Missions, 2015. Available from: https://www.lepr osy.org/ten-steps/.

2 Eze CC, Ekeke N, Alphonsus C, Lehman L, Chukwu JN et al. Effectiveness of self-care interventions for integrated morbidity management of skin neglected tropical diseases in Anambra State, Nigeria. BMC Public Health, 2021; 21: 1748. https://doi.org/10.1186/s12889-021-11729-1. 